\title{
Working-memory training in younger and older adults: training gains, transfer, and maintenance
}

\section{Yvonne Brehmer*, Helena Westerberg and Lars Bäckman}

Aging Research Center, Karolinska Institute, Stockholm, Sweden

\section{Edited by:}

Julia Karbach, Saarland University,

Germany

Reviewed by:

Guido P. H. Band, Leiden University, Netherlands

Kristin E. Flegal, University of

California, Davis, USA

${ }^{*}$ Correspondence:

Yvonne Brehmer, Aging Research

Center, Karolinska Institute,

Gävlegatan 16, 11330 Stockholm,

Sweden.

e-mail:yvonne.brehmer@ki.se
Working memory (WM), a key determinant of many higher-order cognitive functions, declines in old age. Current research attempts to develop process-specific WM training procedures, which may lead to general cognitive improvement. Adaptivity of the training as well as the comparison of training gains to performance changes of an active control group are key factors in evaluating the effectiveness of a specific training program. In the present study, 55 younger adults (20-30 years of age) and 45 older adults (60-70 years of age) received 5 weeks of computerized training on various spatial and verbal WM tasks. Half of the sample received adaptive training (i.e., individually adjusted task difficulty), whereas the other half-worked on the same task material but on a low task difficulty level (active controls). Performance was assessed using criterion, near-transfer, and far-transfer tasks before training, after 5 weeks of intervention, as well as after a 3-month follow-up interval. Results indicate that (a) adaptive training generally led to larger training gains than low-level practice, (b) training and transfer gains were somewhat greater for younger than for older adults in some tasks, but comparable across age groups in other tasks, (c) far-transfer was observed to a test on sustained attention and for a self-rating scale on cognitive functioning in daily life for both young and old, and (d) training gains and transfer effects were maintained across the 3-month follow-up interval across age.

Keywords: working memory, training, aging, transfer, maintenance, active control group

\section{INTRODUCTION}

Working memory (WM), the ability to maintain and manipulate information over short periods of time in the context of concurrent processing or distraction, is a key determinant of several higher-order cognitive functions, such as reasoning, fluid intelligence, problem solving, and language comprehension (Engle, 2002; Borella et al., 2010; Nettelbeck and Burns, 2010). WM functioning declines in late adulthood (Bopp and Verhaeghen, 2005; Payer et al., 2006; Borella et al., 2008) and is considered as one of the main contributing factors of various cognitive impairments in old age (Park et al., 2002). Hence, investigating the possibilities of improving WM functioning in older adults should be highly relevant to everyday cognition in late life. A large number of training studies have investigated the trainability of WM across the lifespan (for reviews, see Klingberg, 2010; Shipstead et al., 2010; Takeuchi et al., 2010; Morrison and Chein, 2011).

The benefit of a cognitive training program can be assessed by the (a) magnitude of gains in the trained tasks, (b) generalization of training effects to other non-trained tasks (transfer), and (c) stability of training and transfer effects across time (Hertzog et al., 2009). Training studies attempting at increasing WM functioning in older adults demonstrate performance gains in trained tasks and closely related non-trained WM tasks (e.g., Mahncke et al., 2006; Buschkuehl et al., 2008; Li et al., 2008; Dahlin et al., 2008a,b; Borella et al., 2010; Schmiedek et al., 2010; Richmond et al., 2011). Findings regarding far-transfer effects in old age are limited. Although generalization of WM training gains to other nontrained task domains (e.g., interference control, fluid intelligence, reasoning, reading comprehension) has been observed in younger adults (e.g., Klingberg et al., 2002; Jaeggi et al., 2008; Dahlin et al., 2008a; Chein and Morrison, 2010; but see Dahlin et al., 2008b; Owen et al., 2010), studies with older adults typically report reduced or non-existent transfer effects (e.g., Buschkuehl et al., 2008; Li et al., 2008; Dahlin et al., 2008a,b; Karbach and Kray, 2009; Borella et al., 2010; Schmiedek et al., 2010; Richmond et al., 2011; Zinke et al., 2012; but see Bherer et al., 2006; Carretti et al., 2007). Regarding the stability of training and transfer effects, there is evidence that older adults are able to maintain performance increments across months (e.g., Mahncke et al., 2006; Li et al., 2008; Dahlin et al., 2008a; Borella et al., 2010; Richmond et al., 2011; but see Buschkuehl et al., 2008).

To investigate the effects of a training program, choice of control group is critical. No-contact (passive) control groups are most commonly used. Here, participants perform pre- and post-training tests to rule out effects based on the fact that the same test is performed twice (i.e., test-retest effects), but participants are not contacted during the training phase (e.g., Li et al., 2008; Dahlin et al., 2008a; Chein and Morrison, 2010; Schmiedek et al., 2010). However, in addition to test-retest effects, the task environment (e.g., performing a specific task regularly, receiving feedback, being challenged with a new testing situation, having contact with test leaders, expectations about performance improvements due to training) might influence performance. Obviously, these influences cannot be eliminated by using a passive control group. Only few studies have used active control groups. In these studies, the controls typically perform activities 
unrelated to the targeted cognitive function (e.g., quizzes, questionnaires of autobiographical memory and well-being, physical activity, watching DVDs), matched on time and effort with the actual training program (e.g., Mahncke et al., 2006; Buschkuehl et al., 2008; Borella et al., 2010; Richmond et al., 2011). However, this procedure has the disadvantage that participants are engaged in quite different tasks that might affect performance differently.

In the present study, we investigated training gains, transfer effects, and 3-month maintenance effects of an intensive computerized WM training in younger and older adults. Experimental as well as control groups worked on the same training software, the only difference being that the experimental groups received adaptive training, while the control groups worked on a constant low task difficulty level. Adaptive training (e.g., individualized adjustment of task difficulty levels) is known to contribute to the efficiency of memory training and to allow individuals to make optimal use of their latent potential (Baltes et al., 1989; Klingberg et al., 2002; Brehmer et al., 2007; Hertzog et al., 2009). We used a process-specific WM training regimen (Park et al., 2007; Morrison and Chein, 2011), with abstract and new stimuli configurations presented at each trial, designed to target domaingeneral WM mechanisms and to minimize the formation and use of domain-specific strategies. Based on previous findings, we expected (a) younger and older adults to benefit from WM training, (b) near-transfer effects to non-trained WM tasks but also some far-transfer to tasks that share similar underlying processes (i.e., attention, reasoning), and (c) maintenance effects for younger as well as older adults across the 3-month time interval for the training gains as well as for potential transfer effects.

\section{METHODS \\ PARTICIPANTS}

Participants were recruited through a newspaper advertisement according to the following inclusion criteria: (a) aged between 20 and 30 years or 60-70 years, (b) healthy and no history of psychiatric or neurological disease, (c) inexperienced to computerized WM training, and (d) access to a PC with Internet connection at home.

Hundred and six adults who fulfilled the inclusion criteria were randomized to either adaptive training or low-level practice (active control) groups. Six persons withdrew from the study after baseline testing (four from the training group and two from the control group) due to technical problems, lack of time, or illness. In the final study sample, 55 younger adults $\left(M_{\text {age }}=\right.$ 26.0, 32 females) and 45 older adults $\left(M_{\text {age }}=63.8,27\right.$ females $)$ completed: (a) cognitive baseline assessment, (b) 5 weeks of intervention, and (c) cognitive post-training assessment. Only one younger adult did not attend the 3-month follow-up assessment due to moving abroad. Hence, valid results of 99 individuals were available for the 3-month follow-up assessment.

The adaptive training and control groups did not differ significantly in age, education, or gender distribution ( $p s>0.80$; see Table 1 for sample descriptives). Regarding completed training days, the two intervention groups did not differ significantly, $F_{(1,96)}=2.8, p=0.10$; however, older adults trained on average 1 day more than younger adults, 24.6 days and 23.5 days, respectively $\left(F_{(1,96)}=10.60, p<0.05, \eta_{p}^{2}=0.10\right)$.

The study was approved by the local ethics committee at the Karolinska Hospital, Stockholm, Sweden. All participants were paid SEK 3000 (approximately 440 USD) for participation.

\section{DESIGN AND PROCEDURES}

This study focuses on age- and intervention-related effects in the cognitive tests assessed before and after 5 weeks of adaptive WM training/low-level practice, as well as at a 3-month follow-up. A more detailed examination of the 5 weeks of intervention is described elsewhere (Brehmer et al., 2009, 2011; Bellander et al., 2011).

\section{Cognitive intervention}

The WM training was implemented using a commercial software product (Cogmed QM), which runs on the participants' PCs at home. Individuals trained for 20-25 days (minimum 20 days) on seven verbal and non-verbal WM tasks. All tasks involved: (1) maintenance of multiple stimuli at the same time, (2) short delays during which the representation of stimuli should be held in WM, and (3) unique sequencing of stimuli order in each trail (for details of the trained tasks, see Bellander et al., 2011).

\section{Adaptive training}

In total, individuals trained on $90 \mathrm{WM}$ trials per day, and needed on average 26 minutes to complete a training session. In the first session, individuals started each task at the same low difficulty level, namely remembering 2 items. Across training, task difficulty was adjusted as a function of individual performance. Specifically, task difficulty was adjusted by increasing/decreasing the number of items individuals had to remember, such that they reached approximately $60 \%$ correct per day for each task (for details about the trained tasks and the adaptive training algorithm, see Cogmed QM; www.cogmed.com, Klingberg et al., 2002). Each training session started at the task difficulty level where the participant ended in the previous session. The test leader provided feedback on the training data once a week via e-mail and controlled

\begin{tabular}{|c|c|c|c|c|}
\hline & \multicolumn{2}{|c|}{ Adaptive training $(n=55)$} & \multicolumn{2}{|c|}{ Low-level practice $(n=45)$} \\
\hline Age & $26.2(2.8)$ & $63.9(3.4)$ & $25.7(3.5)$ & $63.6(3.1)$ \\
\hline Gender distribution & 18 females & 15 females & 14 females & 12 females \\
\hline Years of education & $15(2.6)$ & $15.3(3.4)$ & $15.0(2.8)$ & $15.4(3.5)$ \\
\hline No. of training days & $23.0(2.0)$ & $24.6(1.1)$ & $24.1(1.5)$ & $24.5(1.4)$ \\
\hline
\end{tabular}


the data for potential breaks, interruptions, and unusual performance fluctuations. No problems were observed for any participant.

\section{Low-level practice}

Individuals in the active control groups worked on the same computerized WM program as the adaptive training groups. The differences between the groups were that task difficulty remained constant at the same low starting level for the controls, namely remembering two items. In addition, to adjust for time differences on task due to increased number of items per task in the adaptive training group, the control groups worked on 120 stimuli on each task and day. For motivational reasons, individuals were told to participate in speed training that may have a positive impact on cognitive functioning.

\section{Cognitive assessment at baseline, post-training, and follow-up}

Before and after the 5 weeks of intervention as well as after a 3-month time interval, all individuals were examined with the same set of eight cognitive tests to assess training-related performance gains in the criterion tasks (WM tasks) similar to the ones participants trained for 5 weeks (Span Board forward, Digit Span backward; Wechsler, 1981), near-transfer tasks (Span Board backward, Digit Span forward; Wechsler, 1981), as well as far-transfer tasks (sustained attention, PASAT, Gronwall, 1977; interference control, Stroop, Dodrill, 1978; episodic memory, RAVLT, Lezak, 1983; and non-verbal reasoning, RAVEN, Raven, 1995). In addition, participants completed a self-rating scale for cognitive functioning in daily life (CFQ; Broadbent et al., 1982) at all three measurement occasions. For more details on the tasks, see Klingberg et al. (2002) and Westerberg et al. (2007). Before baseline assessment, participants were randomly assigned to two groups receiving either adaptive training or low-level practice. Participants as well as test leaders were blind as to which experimental group individuals belonged. In addition, individuals' training accounts were locked after post-training assessment. Thus, it was not possible to practice further between post-training and 3-month follow-up assessment.

\section{STATISTICAL ANALYSIS}

\section{Performance gains during training}

This analysis was restricted to the adaptive training groups, due to the fact that the performance of the control groups was fixed at a low-level across the 5 weeks of intervention. All participants in the adaptive training groups completed at least 20 training sessions. Participants' daily performance on the seven different WM tasks was aggregated into one $t$-standardized WM performance score. Weekly WM performance scores were used for analysis (for details on the rationale and implementation of this score, see Bellander et al., 2011 and Brehmer et al., 2011). A mixed repeated-measure ANOVA was conducted with age (young, old) as between-subject factor and time (weeks 1-4) as within-subject factor to investigate performance gains during the training period and potential age differences therein.

\section{Cognitive performance}

One-Way ANOVAs were conducted separately for the eight criterion and transfer tasks and the self-rating scale of cognitive functioning to examine potential baseline differences between the age and intervention groups. To determine differences in trainingrelated changes in the age and intervention groups, mixed repeated measure ANOVAs were conducted with age (young and old) and intervention (adaptive training and low-level practice) as between-subject factors and time (baseline, post-training, and follow-up) as within-subject factor for the eight cognitive tasks and the self-rating scale, respectively. Follow-up analyses were conducted comparing baseline to post-training and post-training to 3-month follow-up assessment. For all analyses, alpha levels were set to 0.05 and effect sizes refer to partial eta-square values.

\section{RESULTS}

\section{PERFORMANCE GAINS DURING TRAINING}

In general, younger adults showed higher performance compared to older adults $\left(F_{(1,53)}=29.19, p<0.001, \eta_{p}^{2}=0.36\right)$. Both adaptive training groups increased their performance across the 4 weeks of training $\left(F_{(3,51)}=121,18, p<0.001, \eta_{\mathrm{p}}^{2}=0.88\right)$; however, younger adults demonstrated larger performance gains than older adults, as indicated by a reliable age $\times$ time interaction, $F_{(3,51)}=2.97, p=0.04, \eta_{\mathrm{p}}^{2}=0.15$. Follow-up analysis revealed that younger adults gained more than older adults from week 1 to week $2\left(F_{(1,53)}=5.85, p=0.02, \eta_{\mathrm{p}}^{2}=0.10\right)$, although the two age groups showed comparable performance gains after week 2 ( $p$ s > 0.05; see Figure 1).

\section{COGNITIVE BASELINE PERFORMANCE}

Older adults' baseline performance was lower in all cognitive tasks and the self-rating scale compared to that of younger adults ( $p s$ $<0.05$ ), the only exceptions being Digit Span forward and backward, where both age groups performed equally well $\left(F_{S}<1\right)$. The two intervention groups did not differ in their baseline performance $(F s<1.3)$ in the self-rating scale or any of the different cognitive tasks apart from the RAVLT, where the low-level practice groups performed better than the adaptive training groups, $F_{(1,96)}=4.53, p=0.04, \eta_{\mathrm{p}}^{2}=0.05$.

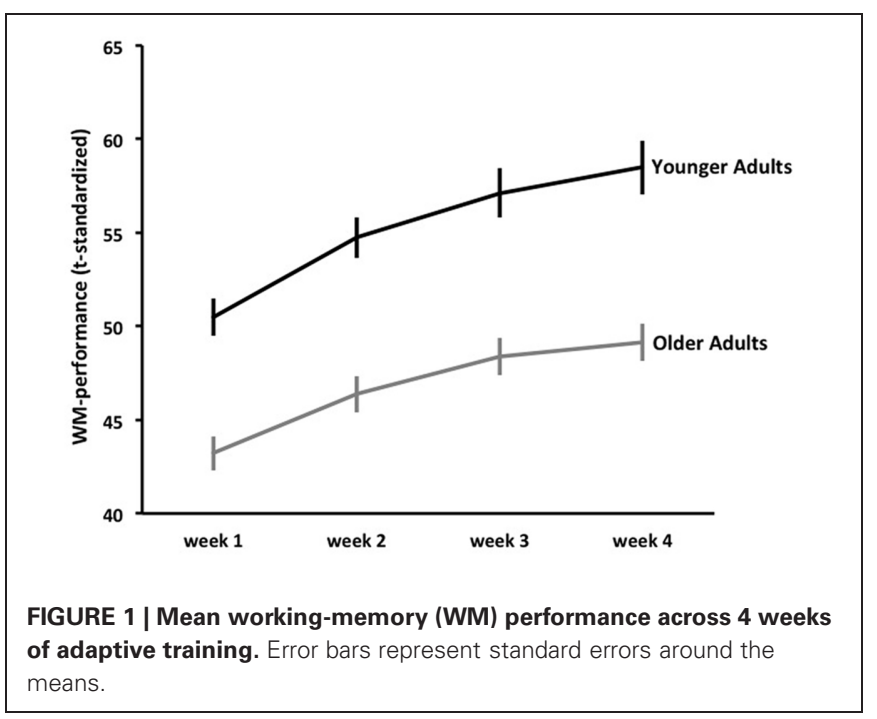




\section{INTERVENTION-RELATED PERFORMANCE CHANGES Criterion tasks}

For both criterion tasks, Span Board forward and Digit Span backward, there were performance increases across time $\left(F_{(2,192)}=39.51, p<0.001, \eta_{p}^{2}=0.29 ; F_{(2,192)}=26.88, p<\right.$ $0.001, \eta_{\mathrm{p}}^{2}=0.22$, respectively). In both tasks, younger adults gained more from training than older adults $\left(F_{(2,192)}=\right.$ $4.24, p=0.02, \eta_{\mathrm{p}}^{2}=0.04 ; F_{(2,192)}=3.78, p=0.02, \eta_{\mathrm{p}}^{2}=0.04$, respectively). In addition, the adaptive training groups showed larger performance increases than the low-level practice groups $\left(F_{(2,192)}=21.85, p<0.001, \eta_{\mathrm{p}}^{2}=0.19 ; F_{(2,192)}=5.70, p<\right.$ $0.001, \eta_{\mathrm{p}}^{2}=0.06$, respectively). For Span Board forward, additional main effects for age $\left(F_{(1,96)}=54.01, p<0.001, \eta_{\mathrm{p}}^{2}=\right.$ $0.36)$ and intervention $\left(F_{(1,96)}=20.16, p<0.001, \eta_{\mathrm{p}}^{2}=0.17\right)$ were observed as well as an age $\times$ intervention interaction $\left(F_{(1,96)}=4.28, p=0.04, \eta_{\mathrm{p}}^{2}=0.04\right)$. The interaction effect reflected that the difference in performance gains between the adaptive training and the low-level practice groups was larger in younger than in older adults (see Table 2 ).

\section{Near-transfer tasks}

For both near-transfer tasks, Span Board backward and Digit Span forward, performance increases across time were again observed $\left(F_{(2,192)}=50.26, p<0.001, \eta_{\mathrm{p}}^{2}=0.34 ; F_{(2,192)}=\right.$ 12.67, $p<0.001, \eta_{\mathrm{p}}^{2}=0.18$, respectively). In both tasks, larger performance gains for the adaptive training than the low-level practice groups were found $\left(F_{(2,192)}=21.32, p<0.001, \eta_{\mathrm{p}}^{2}=\right.$ $0.18 ; F_{(2,192)}=5.11, p=0.01, \eta_{\mathrm{p}}^{2}=0.05$, respectively). In the Span Board backward task, main effects for age and intervention $\left(F_{(1,96)}=81.14, p<0.001, \eta_{\mathrm{p}}^{2}=0.46 ; F_{(1,96)}=23.10, p<\right.$ $0.001, \eta_{\mathrm{p}}^{2}=0.19$, respectively) were observed as well as an age $\times$ intervention interaction $\left(F_{(1,96)}=7.01, p=0.01, \eta_{\mathrm{p}}^{2}=0.07\right)$, reflecting that the difference in performance gains between adaptive training and low-level practice groups was larger in younger than in older adults (see Table 2).

\section{Far-transfer tasks}

For all far cognitive transfer tasks (i.e., PASAT, Stroop, RAVLT, and RAVEN) main effects for age $(p s<0.01)$ were observed indicating higher overall performance for younger than for older adults. Apart from the RAVLT $(F<1)$, all tests showed an additional main effect of time ( $p s<0.01$ ), indicating general performance improvements across time for all groups. More importantly, an intervention $\times$ time interaction was observed for PASAT, $F_{(2,192)}=7.64, p=0.001, \eta_{p}^{2}=0.07$, indicating that the adaptive training groups improved more than the low-level practice groups across the 5 weeks of intervention. No other effects reached significance ( $p s>0.05$, see Table 2 ).

Regarding the self-rating scale on cognitive functioning (CFQ), generally lower memory complaints in younger adults in comparison to older adults were observed $\left(F_{(1,96)}=9.78\right.$, $\left.p=0.002, \eta_{\mathrm{p}}^{2}=0.09\right)$ as well as a general decrease of memory complaints across time $\left(F_{(2,192)}=9.06, p<0.001, \eta_{\mathrm{p}}^{2}=\right.$ $0.86)$. Further, an intervention $\times$ time interaction was obtained, $F_{(2,192)}=3.22, p=0.045, \eta_{\mathrm{p}}^{2}=0.03$, reflecting that the adaptive training groups reduced their memory complaints more than the low-level practice groups across the 5 weeks of intervention (see Table 2).

\section{Maintenance effects}

Further inspection of the time-related effects revealed that in all criterion, near-transfer, as well as in two far-transfer tasks (i.e., RAVEN, PASAT), and the CFQ, the significant main effect of time was based on the difference between baseline and posttraining ( $p s<0.05$ ), whereas post-training and 3-month followup performance did not differ reliably $(p s>0.05)$. Thus, the performance level reached after 5 weeks of intensive WM training was maintained across 3-months. Further, for RAVLT no performance change across time was observed; for Stroop, time on task decreases were observed after the 5 weeks of intervention as well as at the 3-month follow-up $\left(F_{(1,96)}=43.24, p<0.001\right.$, $\eta_{\mathrm{p}}^{2}=0.31 ; F_{(1,96)}=13.06, p<0.001, \eta_{\mathrm{p}}^{2}=0.12$, respectively). For the intervention $x$ time interaction in the criterion and neartransfer tasks, as well as for PASAT and CFQ, follow-up analyses again revealed a significant difference between baseline and posttraining ( $p s<0.05$ ), but not between post-training and follow-up ( $p s>0.05$ ), indicating that the difference between adaptive training and low-level practice groups was maintained across the 3-month follow-up interval.

\section{DISCUSSION}

The present study investigated the effects of 5 weeks of intensive domain-general adaptive WM training in comparison to low-level practice in younger and older adults. Performance was assessed using criterion, near-transfer, and far-transfer tasks before training, after 5 weeks of intervention, as well as after a 3 -month time interval. Younger as well as older adults gained considerably from adaptive WM training. Although younger adults showed larger training gains than older adults during the first week, both age groups gained similarly after the second week. Both younger and older adults gained more in some criterion and non-trained WM tasks (Digit Span) in comparison to controls receiving low-level practice, although we observed larger gains and transfer effects for the young in other criterion and near-transfer tasks (Span Board). Regarding far-transfer, similar performance improvements for the adaptive training as well as the active control groups were observed for tests of interference control (Stroop) and reasoning (RAVEN). These findings demonstrate general test-retest effects. More interestingly, both younger and older adults receiving adaptive training showed larger performance gains in a test measuring sustained attention (PASAT) and reported less memory complaints (CFQ) after the 5 weeks of intervention than the controls. Further, the observed training gains and transfer effects were maintained across a 3-month time interval. The same set of eight cognitive tests to assess training-related performance gains and transfer effects were used at the three assessment occasions (i.e., baseline, posttest, 3-month follow-up). Thus, potential retest influences on the observed performance changes cannot be excluded. However, this possibility does not affect the observed training and transfer effects. This is so because by including an active control group, test-retest effects were accounted for, ensuring that the additional performance changes resulted from the adaptive WM training. 


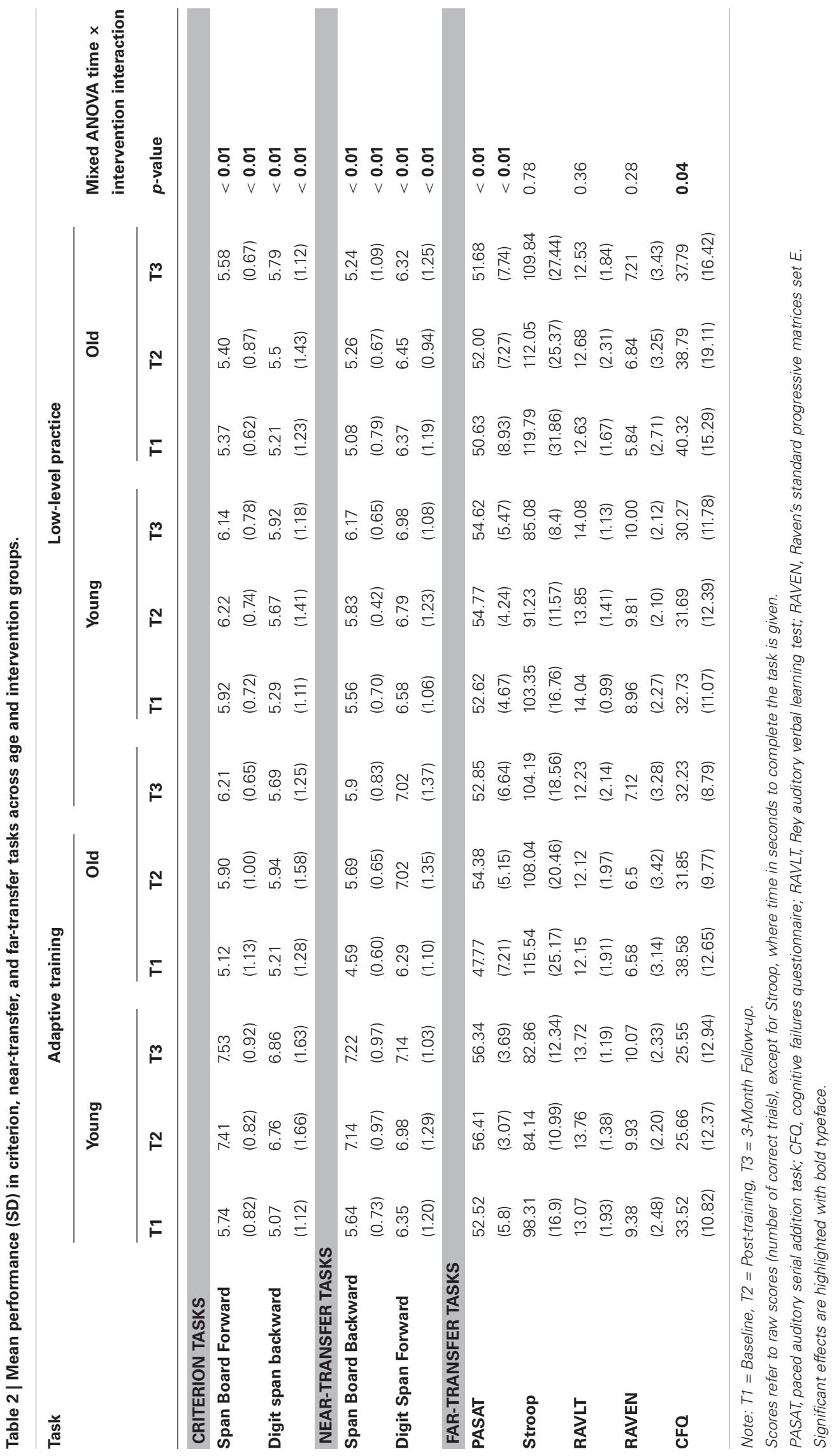


We used a domain-general computerized WM training paradigm, which has been employed in previous studies with children, younger adults as well as persons with acquired brain lesions (Klingberg et al., 2002, 2005; Olesen et al., 2004; Westerberg et al., 2007; Holmes et al., 2009; Thorell et al., 2009; Jolles et al., 2010). These studies consistently observe near-transfer effects to non-trained WM tasks and often far-transfer effects to tests of attention, interference control, and reasoning. Our findings are in line with this previous work and other training studies in the aging domain regarding near-transfer effects to non-trained WM tasks and far-transfer to sustained attention (e.g., Mahncke et al., 2006; Mozolic et al., 2010, 2011; Richmond et al., 2011). Our expectations regarding transfer effects relied on the assumption that training and transfer tasks have to tap on similar underlying processes required for successful performance (Thorndike and Woodworth, 1901). Our domain-general WM training included processes like attention control, gating the flow of information, reducing interference while requiring maintenance of stored information, and rapid shifting between encoding and retrieval demands. Hence, the transfer of our WM intervention to the PASAT suggests that the training also improved attentional focusing.

Most often transfer effects in older adults are difficult to demonstrate and, when observed, they are reduced compared to younger adults (Buschkuehl et al., 2008; Li et al., 2008; Dahlin et al., 2008a,b; Karbach and Kray, 2009; Schmiedek et al., 2010; Richmond et al., 2011; Zinke et al., 2012). In accordance with this research, we observed larger training gains for younger than for older adults in one of the two criterion tasks (Span Board Forward) and one of the near-transfer tasks (Span Board Backward). However, younger and older adults did not differ in training and transfer effects in the two Digit Span tasks. It remains unclear why we observed age differences in the magnitude of gains for the Span Board tasks, but not for the Digit Span tasks. With regard to Digit Span, previous studies have not observed any improvements after WM training in older adults (Buschkuehl et al., 2008; Li et al., 2008; Dahlin et al., 2008b; Richmond et al., 2011). Future training studies should consider the approach suggested by Lövden et al. (2010), using established hierarchical structures of cognitive abilities instead of single tests (see also Schmiedek et al., 2010) to assess training and transfer effects. However, in our study performance gains on the PASAT as well as for the CFQ were also comparable in younger and older adults. These findings are in line with some previous studies (e.g., Bherer et al., 2006; Carretti et al., 2007) suggesting that our training paradigm is a sensitive means to detecting cognitive plasticity even in older individuals. This might reflect the adaptive algorithm used in our study, which provides a challenging task situation for the participants, the variety of verbal and non-verbal tasks used in the training program (i.e., domain-general instead

\section{REFERENCES}

Baltes, P. B., Sowarka, D., and Kliegl, R. (1989). Cognitive training research on fluid intelligence in old age: what can older adults achieve by themselves? Psychol. Aging 4, 217-221.
Bellander, M., Brehmer, Y., Westerberg, H., Karlsson, S., Furth, D., Bergman, O., Eriksson, E., and Bäckman, L. (2011). Preliminary evidence that allelic variation in the LMX1A gene influences training-related

of process-specific), as well as the structure and appearance of the training program.

Younger as well as older adults reported less memory complains (CFQ) after adaptive WM training in comparison to participants in the active control groups. This was the case even though participants were blind to group assignment; hence placebo/expectancy effects would be an unlikely explanation for this finding. These self-reported cognitive improvements may have important implications for everyday cognitive functioning and should be investigated further in future studies (see also Richmond et al., 2011).

The nature of our control group needs to be highlighted. To our knowledge, this is the first study in the aging domain using an active control group where individuals worked on the same task material as the experimental group, the only difference being that task difficulty was fixed at a low-level. The use of such an active control group (as opposed to no-contact controls or active controls performing different tasks) provides a conservative assessment of training effects, because the influence of various unspecific factors (e.g., stimulus-response mappings, motivation, test familiarity, performance anxiety, expectations) is attenuated (Zehnder et al., 2009; Shipstead et al., 2010). Although no direct measure of motivation was included in the present study, the uniformly high number of training sessions (i.e., 24.6 for younger adults and 23.5 for older adults) for the experimental and control groups (a minimum of only 20 sessions was required), speaks for highly engaged and committed participants in both training groups.

This conservative assessment of training and transfer gains strengthens the impact of our observed effects and suggests caution in comparing our results with other studies using passive control groups, especially in light of the fact that we observed comparable performance improvements for adaptive training groups and the controls for some of the transfer tasks (interference control, reasoning). To be able to disentangle different performanceinfluencing factors and to make assumptions about the value of adaptive training over low-level practice, future studies should include both active and passive (no-contact) control groups.

\section{ACKNOWLEDGMENTS}

Yvonne Brehmer was funded by the Swedish Research Council for Working Life and Social Research through a FLARE postdoctoral grant announced by ERA-AGE. Helena Westerberg was supported by grants from the Swedish Research Council and Hjärnfonden. Lars Bäckman was supported by grants from the Swedish Research Council and Swedish Brain Power, an Alexander von Humboldt Research Award, and by a donation from the af Jochnick Foundation. The authors thank CogMed for allowing them access to the training program and David Söderman and Nicolas D'Hondt for assistance in data collection.

working memory improvement. Neuropsychologia 49, 1938-1942.

Bherer, L., Kramer, A. F., Peterson, M. S., Colcombe, S., Erickson, K., and Becic, E. (2006). Testing the limits of cognitive plasticity in older adults: application to attentional control. Acta Psychol. (Amst.) 123, 261-278.

Bopp, K. L., and Verhaeghen, P. (2005). Aging and verbal memory span: a meta-analysis. J. Gerontol. B Psychol. Sci. Soc. Sci. 60, P223-P233. 
Borella, E., Carretti, B., and De Beni, R. (2008). Working memory and inhibition across the adult life-span. Acta Psychol. (Amst.) 128, 33-44.

Borella, E., Carretti, B., Riboldi, F., and De Beni, R. (2010). Working memory training in older adults: evidence of transfer and maintenance effects. Psychol. Aging 25, 767-778.

Brehmer, Y., Li, S. C., Müller, V., Von Oertzen, T., and Lindenberger, U. (2007). Memory plasticity across the life span: uncovering children's latent potential. Dev. Psychol. 43, 465-478.

Brehmer, Y., Rieckmann, A., Bellander, M., Westerberg, H., Fischer, H., and Bäckman, L. (2011). Neural correlates of training-related working-memory gains in old age. Neuroimage 58, 1110-1120.

Brehmer, Y., Westerberg, H., Bellander, M., Furth, D., Karlsson, S., and Bäckman, L. (2009). Working memory plasticity modulated by dopamine transporter genotype. Neurosci. Lett. 467, 117-120.

Broadbent, D. F., Cooper, P. F., Fitzgerald, P., and Parkes, K. R. (1982). The cognitive failures questionnaire (CFQ) and its correlates. Br. J. Clin. Psychol. 21, 1-16.

Buschkuehl, M., Jaeggi, S. M., Hutchison, S., Perrig-Chiello, P., Dapp, C., Muller, M., Breil, F., Hoppeler, H., and Perrig, W. J. (2008). Impact of working memory training on memory performance in old-old adults. Psychol. Aging 23, 743-753.

Carretti, B., Borella, E., and De Beni, R. (2007). Does strategic memory training improve the working memory performance of younger and older adults? Exp. Psychol. 54, 311-320.

Chein, J. M., and Morrison, A. B. (2010). Expanding the mind's workspace: training and transfer effects with a complex working memory span task. Psychon. Bull. Rev. 17, 193-199.

Dahlin, E., Neely, A. S., Larsson, A., Bäckman, L., and Nyberg, L. (2008a). Transfer of learning after updating training mediated by the striatum. Science 320, 1510-1512.

Dahlin, E., Nyberg, L., Bäckman, L., and Neely, A. S. (2008b). Plasticity of executive functioning in young and older adults: immediate training gains, transfer, and long-term maintenance. Psychol. Aging 23, 720-730.

Dodrill, C. (1978). Neuropsychological battery for epilepsy. Epilepsia 19, 611-623.

Engle, R. W. (2002). Working memory capacity as executive attention. Curr. Dir. Psychol. Sci. 11, 19-23.
Gronwall, D. (1977). Paced auditory serial-addition task - Measure of recovery from concussion. Percept. Mot. Skills 44, 367-373.

Hertzog, C., Kramer, A. F., Wilson, R. S., and Lindenberger, U. (2009). Enrichment effects on adult cognitive development. Psychol. Sci. Public Interest 9, 1-65.

Holmes, J., Gathercole, S. E., and Dunning, D. L. (2009). Adaptive training leads to sustained enhancement of poor working memory in children. Dev. Sci. 12, F9-F15.

Jaeggi, S. M., Buschkuehl, M., Jonides, J., and Perrig, W. J. (2008). Improving fluid intelligence with training on working memory. Proc. Natl. Acad. Sci. U.S.A. 105, 6829-6833.

Jolles, D. D., Grol, M. J., Van Buchem, M. A., Rombouts, S. A., and Crone, E. A. (2010). Practice effects in the brain: changes in cerebral activation after working memory practice depend on task demands. Neuroimage 52, 658-668.

Karbach, J., and Kray, J. (2009). How useful is executive control training? Age differences in near and far transfer of task-switching training. Dev. Sci. 12, 978-990.

Klingberg, T. (2010). Training and plasticity of working memory. Trends Cogn. Sci. 14, 317-324.

Klingberg, T., Fernell, E., Olesen, P. J., Johnson, M., Gustafsson, P., Dahlstrom, K., Gillberg, C. G., Forssberg, H., and Westerberg, $\mathrm{H}$. (2005). Computerized training of working memory in children with ADHD-a randomized, controlled trial. J. Am. Acad. Child Adolesc. Psychiatry 44, 177-186.

Klingberg, T., Forssberg, H., and Westerberg, H. (2002). Training of working memory in children with ADHD. J. Clin. Exp. Neuropsychol. 24, 781-791.

Lezak, M. D. (1983). Neuropsychological Assessment. New York, NY: Oxford U.P.

Li, S. C., Schmiedek, F., Huxhold, O., Rocke, C., Smith, J., and Lindenberger, U. (2008). Working memory plasticity in old age: practice gain, transfer, and maintenance. Psychol. Aging 23, 731-742.

Lövden, M., Bäckman, L., Lindenberger, U., Schaefer, S., and Schmiedek, F. (2010). A theoretical framework for the study of adult cognitive plasticity. Psychol. Bull. 136, 659-676.

Mahncke, H. W., Connor, B. B., Appelman, J., Ahsanuddin, O. N., Hardy, J. L., Wood, R. A., Joyce, N. M., Boniske, T., Atkins, S. M., and Merzenich, M. M. (2006). Memory enhancement in healthy older adults using a brain plasticity-based training program: a randomized, controlled study. Proc. Natl. Acad. Sci. U.S.A. 103, 12523-12528.

Morrison, A. B., and Chein, J. M. (2011). Does working memory training work? The promise and challenges of enhancing cognition by training working memory. Psychon. Bull. Rev. 18, 46-60.

Mozolic, J. L., Hayasaka, S., and Laurienti, P. J. (2010). A cognitive training intervention increases resting cerebral blood flow in healthy older adults. Front. Hum. Neurosci. 4:16. doi: 10.3389/neuro.09.016.2010

Mozolic, J. L., Long, A. B., Morgan, A. R., Rawley-Payne, M., and Laurienti, P. J. (2011). A cognitive training intervention improves modality-specific attention in a randomized controlled trial of healthy older adults. Neurobiol. Aging 32, 655-668.

Nettelbeck, T., and Burns, N. R. (2010). Processing speed, working memory and reasoning ability from childhood to old age. Pers. Individ. Dif. 48, 379-384.

Olesen, P. J., Westerberg, H., and Klingberg, T. (2004). Increased prefrontal and parietal activity after training of working memory. Nat. Neurosci. 7, 75-79.

Owen, A. M., Hampshire, A., Grahn, J. A., Stenton, R., Dajani, S., Burns, A. S., Howard, R. J., and Ballard, C. G. (2010). Putting brain training to the test. Nature 465, 775-778.

Park, D. C., Lautenschlager, G., Hedden, T., Davidson, N. S., Smith, A. D., and Smith, P. K. (2002). Models of visuospatial and verbal memory across the adult life span. Psychol. Aging 17, 299-320.

Park, S., Kim, M. S., and Chun, M. M. (2007). Concurrent working memory load can facilitate selective attention: evidence for specialized load. J. Exp. Psychol. Hum. Percept. Perform. 33, 1062-1075.

Payer, D., Marshuetz, C., Sutton, B. Hebrank, A., Welsh, R. C., and Park, D. C. (2006). Decreased neural specialization in old adults on a working memory task. Neuroreport 17, 487-491.

Raven, J. C. (1995). Standard Progressive Matrices Sets A-E. Oxford, UK: Oxford Psychologists Press Ltd.

Richmond, L. L., Morrison, A. B., Chein, J. M., and Olson, I. R. (2011). Working memory training and transfer in older adults. Psychol. Aging 26, 813-822.

Schmiedek, F., Lovden, M., and Lindenberger, U. (2010). Hundred days of cognitive training enhance broad cognitive abilities in adulthood: findings from the COGITO study. Front. Aging Neurosci. 2:27. doi: 10.3389/fnagi.2010.00027

Shipstead, Z., Redick, T. S., and Engle, R. W. (2010). Does working memory training generalize? Psychol. Belg. 50, 245-276.

Takeuchi, H., Taki, Y., and Kawashima, R. (2010). Effects of working memory training on cognitive functions and neural systems. Rev. Neurosci. 21, 427-449.

Thorell, L. B., Lindqvist, S., Nutley, S. B., Bohlin, G., and Klingberg, T. (2009). Training and transfer effects of executive functions in preschool children. Dev. Sci. 12, 106-113.

Thorndike, E. L., and Woodworth, R. S. (1901). The influence of improvement in one mental function upon the efficiency of other functions. Psychol. Rev. 8, 247-261.

Wechsler, D. (1981). WAIS-R Manual. New York, NY: The Psychological Corporation.

Westerberg, H., Jacobaeus, H., Hirvikoski, T., Clevberger, P., Ostensson, M. L., Bartfai, A., and Klingberg, T. (2007). Computerized working memory training after stroke - A pilot study. Brain Inj. 21, 21-29.

Zehnder, F., Martin, M., Altgassen, M., and Clare, L. (2009). Memory training effects in old age as markers of plasticity: a meta-analysis. Restor. Neurol. Neurosci. 27, 507-520.

Zinke, K., Zeintl, M., Eschen, A., Herzog, C., and Kliegel, M. (2012). Potentials and limits of plasticity induced by working memory training in old-old age. Gerontology 58, 79-87.

Conflict of Interest Statement: The authors declare that the research was conducted in the absence of any commercial or financial relationships that could be construed as a potential conflict of interest.

Received: 26 January 2012; paper pending published: 05 February 2012; accepted: 11 March 2012; published online: 27 March 2012.

Citation: Brehmer Y, Westerberg $H$ and Bäckman L (2012) Working-memory training in younger and older adults: training gains, transfer, and maintenance. Front. Hum. Neurosci. 6:63. doi: 10.3389/fnhum.2012.00063

Copyright (c) 2012 Brehmer, Westerberg and Bäckman. This is an open-access article distributed under the terms of the Creative Commons Attribution Non Commercial License, which permits noncommercial use, distribution, and reproduction in other forums, provided the original authors and source are credited. 